University of Tennessee at Chattanooga

UTC Scholar

ReSEARCH Dialogues Conference Proceedings

ReSEARCH Dialogues Conference Proceedings

Apr 15th, 1:00 PM - 3:00 PM

\title{
MARS: Machine learning-based Adaptable and Robust network management for Software-defined networks
}

\author{
Ilker Ozcelik \\ University of Tennessee at Chattanooga \\ Farah Kandah \\ University of Tennessee at Chattanooga \\ Brennan Huber \\ University of Tennessee at Chattanooga
}

Follow this and additional works at: https://scholar.utc.edu/research-dialogues

\begin{abstract}
Recommended Citation
Ozcelik, Ilker; Kandah, Farah; and Huber, Brennan, "MARS: Machine learning-based Adaptable and Robust network management for Software-defined networks". ReSEARCH Dialogues Conference proceedings. https://scholar.utc.edu/research-dialogues/2020/day2_presentations/55.
\end{abstract}

This presentations is brought to you for free and open access by the Conferences and Events at UTC Scholar. It has been accepted for inclusion in ReSEARCH Dialogues Conference Proceedings by an authorized administrator of UTC Scholar. For more information, please contact scholar@utc.edu. 
MARS: Machine Learning Based Adaptable and Robust Network Management for Software-defined Networks

Farah Kandah ${ }^{6}$, Ilker Ozcelik, Brennan Huber ${ }^{\beta}$ SimCenter ${ }^{\alpha}$

College of Engineering and Computer Science ${ }^{b}$ 


\section{Bio - Ilker Ozcelik, PhD}

$\square$ M.S., Syracuse University, Thesis: Voice Activity Detection using Stochastic Resonance, 2010, Electrical Engineering

$\square$ Ph.D., Clemson University, Dissertation: Denial of Service Attack Detection and Mitigation, 2015, Electrical Engineering

๑ Recep Tayyip Erdogan University, August 2016 - Present, Assistant Professor

$\square$ Recep Tayyip Erdogan University, 2017 - 2019, Department Chair of Information Systems Engineering

曰 SimCenter, University of Tennessee at Chattanooga, September 2019 -- Present, Visiting Scholar and Research Associate 


\section{Outline}

- Motivation \& Problem Statement

Existing Solutions in the Literature

$\square$ MARS

$\square$ Road Map

$\square$ System Setup

Q\&A 


\section{Motivation \& Problem Statement}

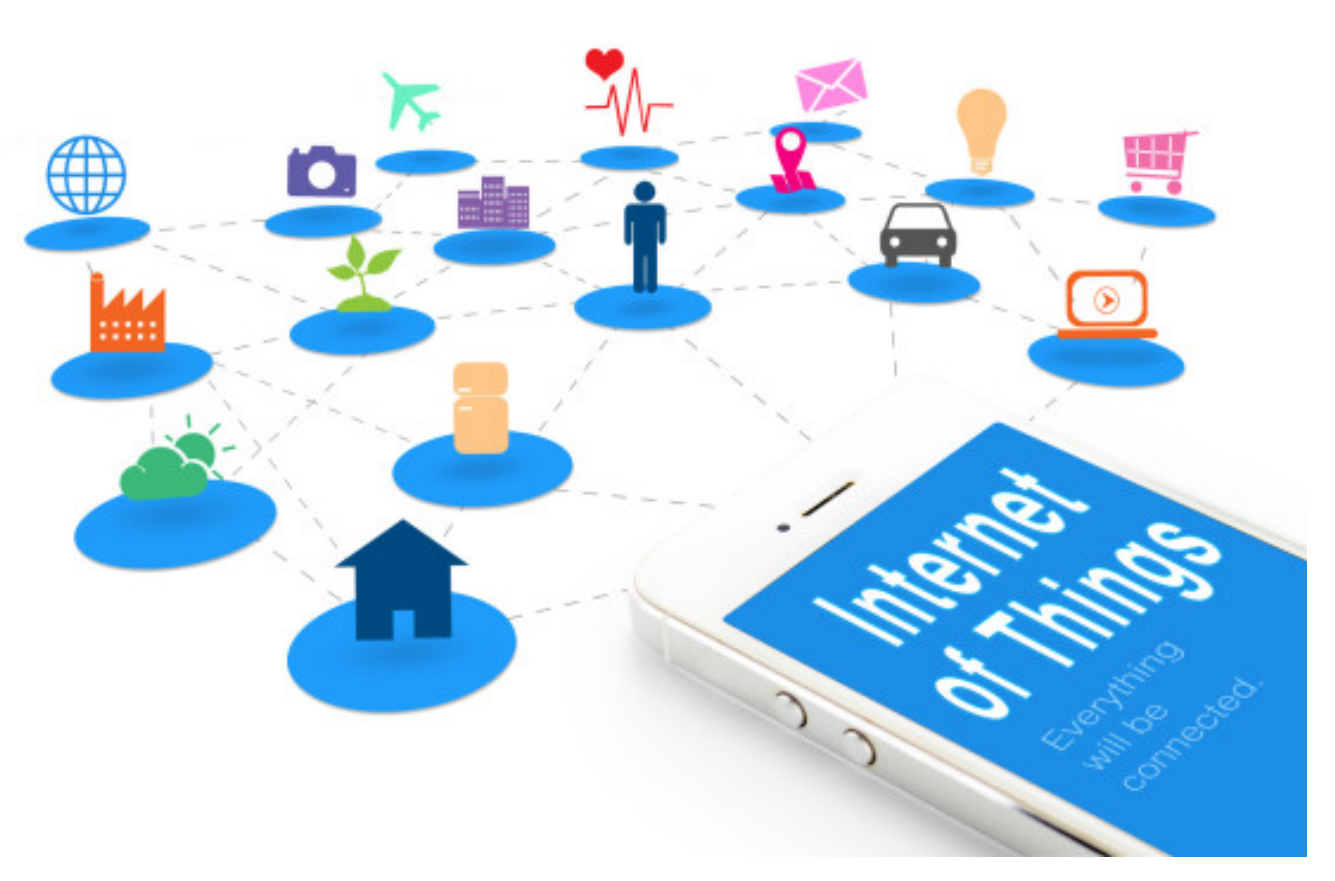

$\square$ Traditional networks were designed to scale fast

- They are harder to monitor and manage

$\square$ Internet of Things (IOT) has caused an increase in the number of nodes

$\square$ Mobile technologies caused constant topology changes on networks

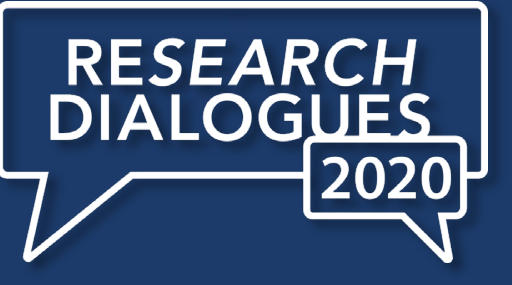




\section{Motivation \& Problem Statement}
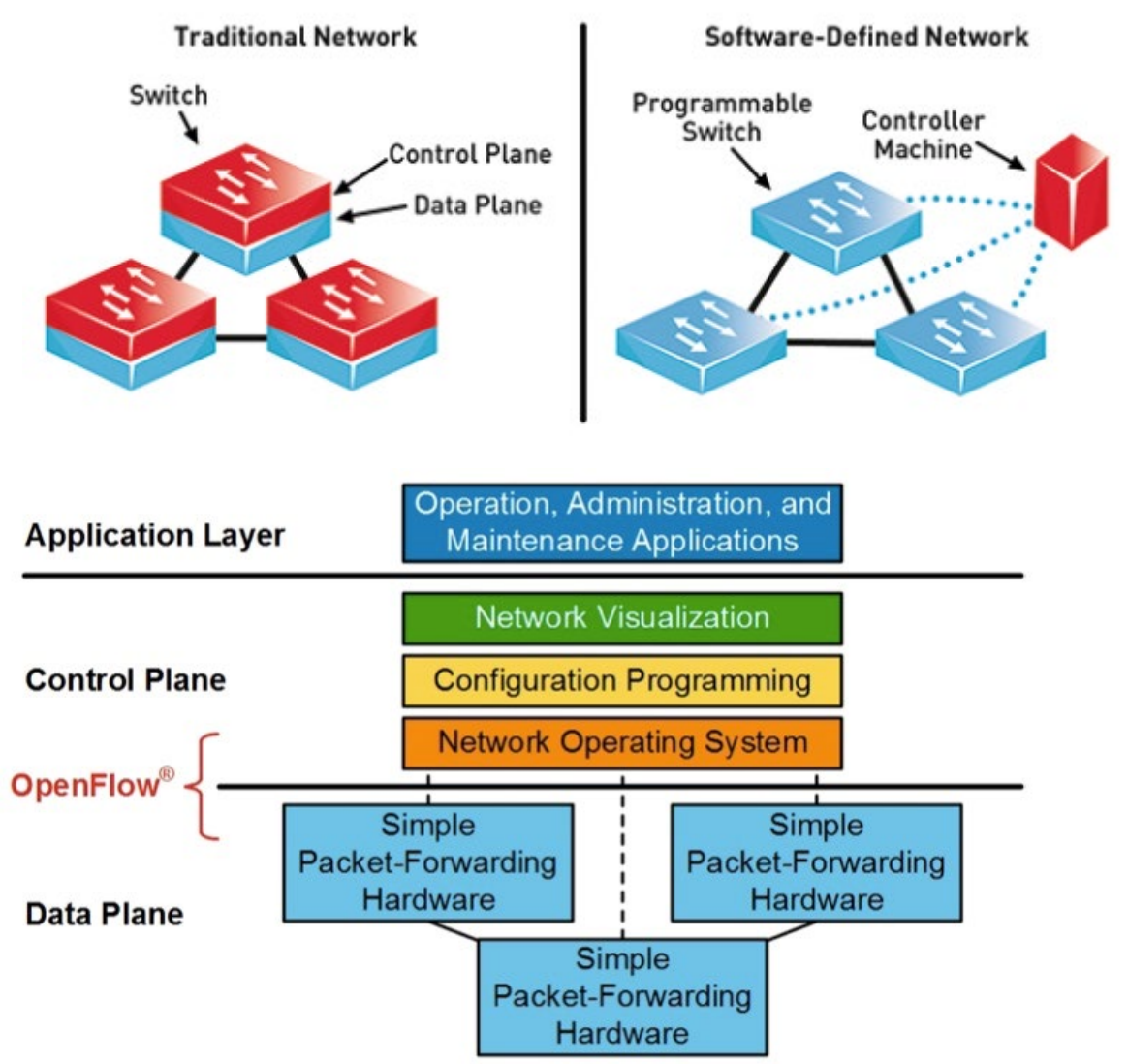

- Software Defined Networking (SDN) addresses network monitoring and management problems

- It has scalability and single point of failure problems

$\square$ We need networks that;

- Are easy to monitor and manage

- Can adapt topology changes to utilize resources efficiently and avoid failures autonomously

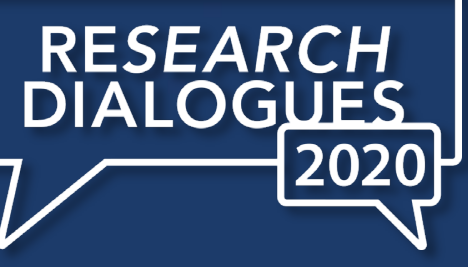




\section{Literature}

$\square$ Scalable and fault tolerant SDN

- Elastic Distributed controller architecture

- Pose as single controller

- Dynamically grow and shrink

- Migrate switches between controllers to loadbalance

- Coordinating multiple control domains

- Controllers exchange status information with each other

- Each controller has global view

- Aim to minimize flow setup time by adjusting number of controllers and delegating the switches

- Utilize controller capacity efficiently

- Determining and eliminating overloading controllers

- Hierarchy-based network architecture

- Domains are controlled by their controllers

- Controllers rely on a broker (Higher level controller)

- Local controller does not have global view
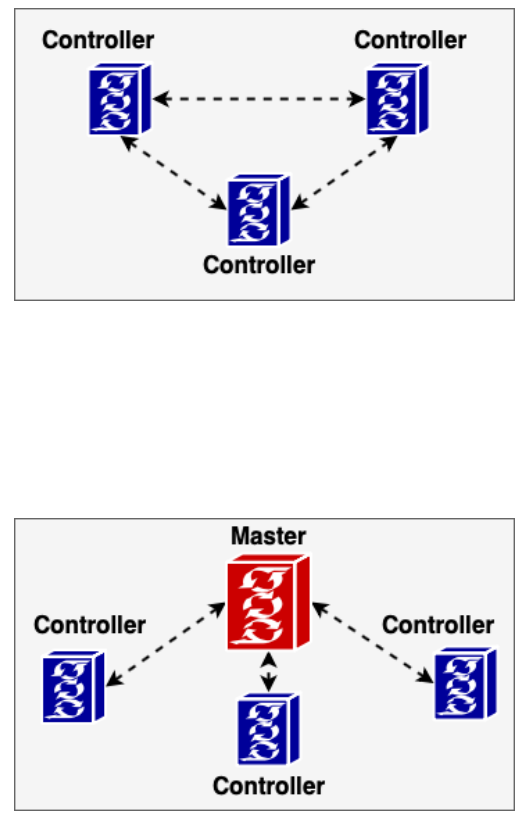


\section{MARS Design}

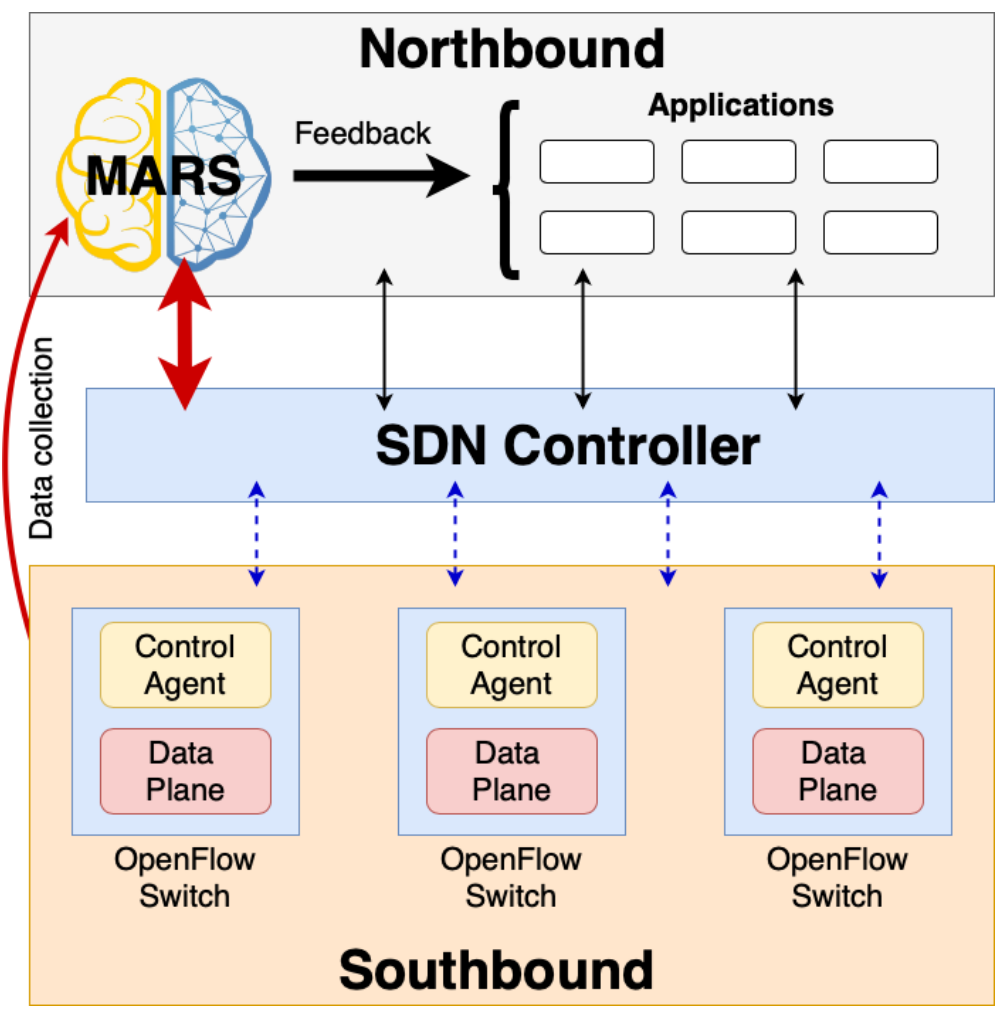

$\square$ Data mining and Machine Learning techniques to understand the network dynamics

$\square$ Network control plane that can autonomously adopt to network changes

- A control plane architecture that can utilize network resources efficiently and can scale

$\square$ An elastic control plane to prevent single point of failure 


\section{Road Map}

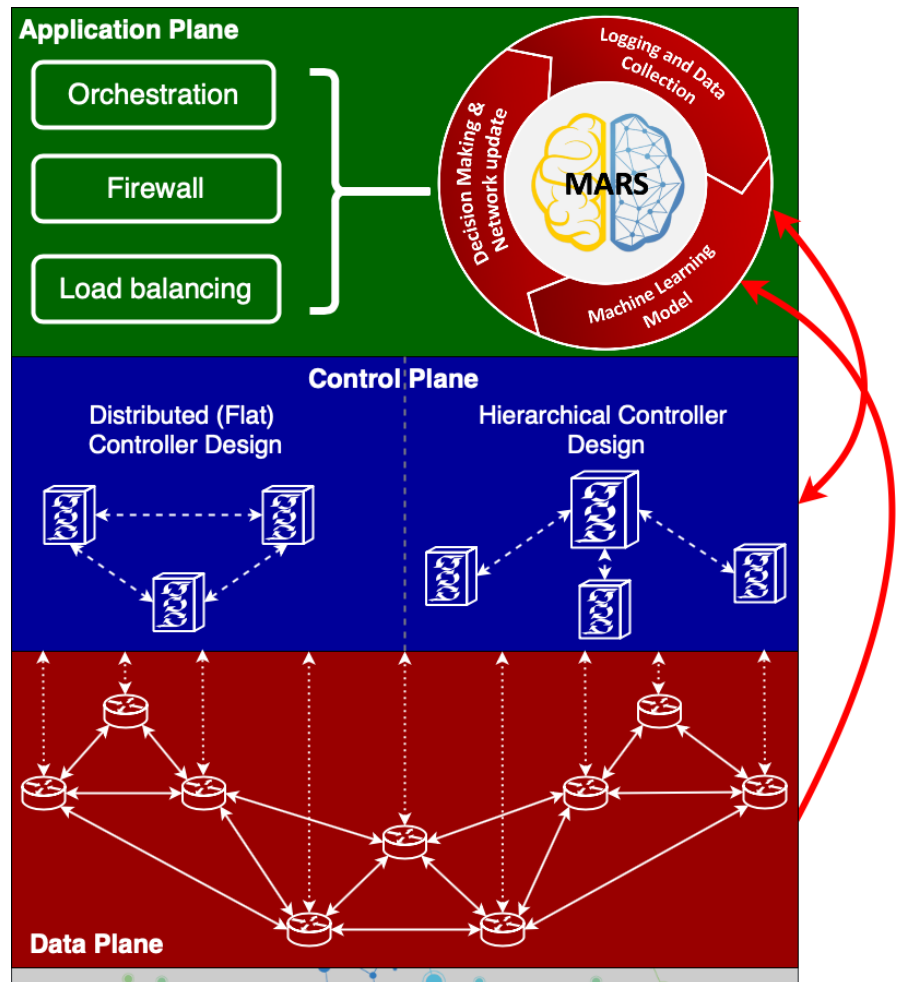

Data Collection

- Controller Level

- Network Level

- Switch stats

- Port Stats

- Pattern Recognition \& Learning

$\square$ Decision Making \& Update

๑ Controller Plane Topology

- Distributed Flat

- Hierarchical

๑ Performance Metric

- Number of flow requests handled per second

- Flow setup latency

Internet of Things

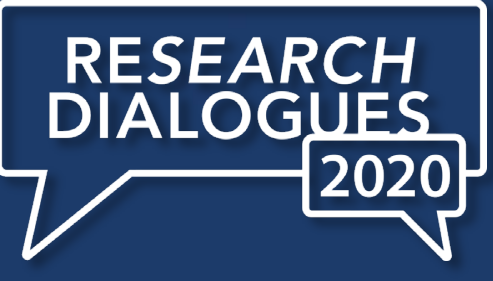




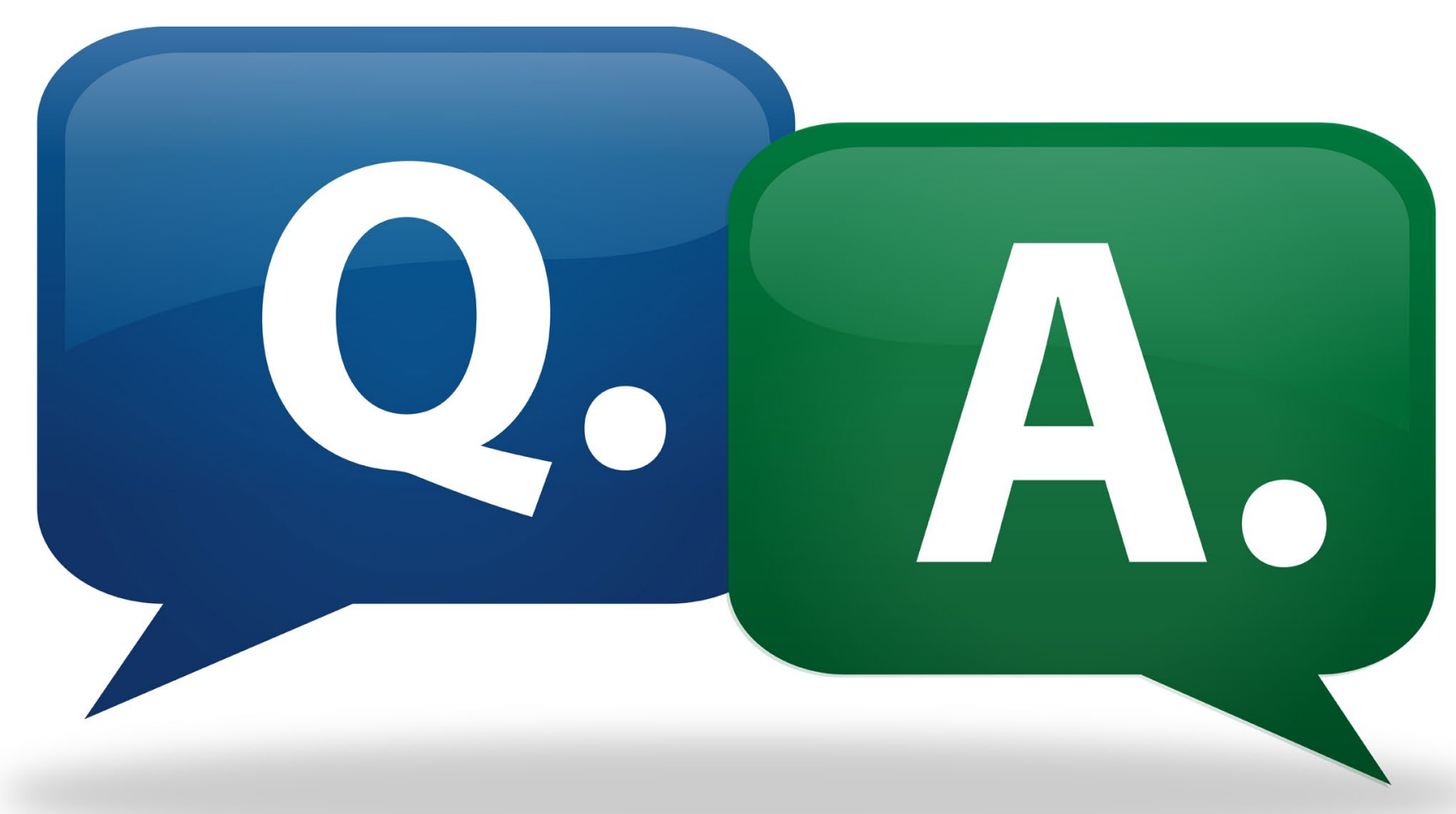

Ilker Ozcelik, PhD SimCenter

ilker-ozcelik@utc.edu ozcelikilker@ieee.org

RESEARCH

DIALOGUES

2020 